\title{
Association of Chronic Immune-Mediated Diarrhea and Colitis With Favorable Cancer Response
}

\author{
Fangwen Zou, MD1,2; Hamzah Abu-Sbeih, MD³; Weijie Ma, MD2,4; Yuanzun Peng, BA5; Wei Qiao, PhD6; \\ Jianbo Wang, MD, PhD; Amishi Y. Shah, MD7; Isabella C. Glitza Oliva, MD, PhD; ; Sarina A. Piha-Paul, MD; \\ John A. Thompson, MD ${ }^{10}$; Hao Chi Zhang, MD²; Anusha S. Thomas, $\mathrm{MD}^{2, *}$; and Yinghong Wang, MD, PhD ${ }^{2, \star}$
}

\begin{abstract}
Background: Immune-mediated diarrhea and colitis (IMDC) is a common immune-related adverse effect related to immune checkpoint inhibitors. We aimed to identify risk factors for chronic IMDC and its prognostic value in cancer outcomes. Methods: We retrospectively collected data on patients with a diagnosis of IMDC between January 2018 and October 2019 and grouped them based on disease duration into acute ( $\leq 3$ months) and chronic ( $>3$ months) categories. A logistic regression model and the Kaplan-Meier method with log-rank tests were used for biostatistical analysis. Results: In our sample of 88 patients, 43 were in the chronic group and 45 were in the acute group. Genitourinary cancer and melanoma accounted for $70 \%$ of malignancies. PD-1/L1 monotherapy (52\%) was the more frequently used regimen. We showed that chronic IMDC was associated with proton pump inhibitor use (odds ratio [OR], 3.96; $P=.026)$, long duration of IMDC symptoms $(O R, 1.05 ; P<.001)$ and hospitalization (OR, 1.07; $P=.043)$, a histologic feature of chronic active colitis (OR, 4.8; $P=.025)$ or microscopic colitis (OR, 5.0; $P=.045)$, and delayed introduction of selective immunosuppressive therapy (infliximab/vedolizumab; OR, 1.06; $P=.047$ ). Chronic IMDC also reflected a better cancer response to immune checkpoint inhibitors $(30 \%$ vs $51 \% ; P=.002)$ and was accompanied by improved overall survival $(P=.035)$. Similarly, higher doses of selective immunosuppressive therapy were associated with better overall survival $(P=.018)$. Conclusions: Chronic IMDC can develop among patients with a more aggressive disease course and chronic features on colon histology. It likely reflects a prolonged immune checkpoint inhibitor effect and is associated with better cancer outcome and overall survival.
\end{abstract}

J Natl Compr Canc Netw 2021;19(6):700-708 doi: $10.6004 /$ jnccn.2020.7647

\footnotetext{
${ }^{1}$ Department of Oncology, The Second Xiangya Hospital of Central South University, Changsha, Hunan, PR China; ${ }^{2}$ Department of Gastroenterology, Hepatology, and Nutrition, The University of Texas MD Anderson Cancer Center, Houston, Texas; ${ }^{3}$ Department of Internal Medicine, University of Missouri, Kansas City, Missouri; ${ }^{4}$ Department of Hepatobiliary and Pancreatic Surgery, Zhongnan Hospital of Wuhan University, Wuhan, China; ${ }^{5}$ Department of Biosciences, Rice University, Houston, Texas; ${ }^{6}$ Department of Biostatistics, ${ }^{7}$ Department of Genitourinary Medical Oncology, ${ }^{8}$ Department of Melanoma Medical Oncology, and ${ }^{9}$ Department of Investigational Cancer Therapeutics, The University of Texas MD Anderson Cancer Center, Houston, Texas; and

${ }^{10}$ Department of Medicine, University of Washington, Fred Hutchinson Cancer Research Center, Seattle, Washington.
}

*These authors contributed equally as senior authors.

\section{Background}

Immune checkpoint inhibitor (ICI) therapy significantly improves cancer outcomes by targeting CLTA- 4 or PD-1/ $\mathrm{L} 1$ and boosting T-cell-mediated immune responses and antitumor effects. ${ }^{1,2}$ ICIs are widely used as first-line therapy for many advanced malignancies, with remarkable cancer response and overall survival (OS).,3

However, ICI therapy predisposes patients to an imbalance of normal immune tolerance and bears the risk of immune-related adverse effects (irAEs). Immunemediated diarrhea and colitis (IMDC), the second most common irAE, limits the use of ICIs. ${ }^{5}$ The incidence and severity of IMDC vary depending on the ICI regimen. Severe forms are attributed to anti-CTLA-4-based regimens, ${ }^{6}$ and clinical symptoms range from self-limited diarrhea to severe life-threatening diarrhea, bleeding, colonic dilatation, and bowel perforation. ${ }^{7}$ Multiple studies have shown an association between IMDC and improved OS in patients with cancer, with diarrhea being an independent predictor of improved OS. ${ }^{8}$

For management of IMDC, oncologic guidelines recommend corticosteroids as first-line therapy, with selective immunosuppressive therapy (SIT) as secondline treatment for patients with refractory cases. Recent studies report benefits from early introduction and higher doses of SIT to the IMDC disease course and outcome. ${ }^{9,10}$ However, a subgroup of patients continues to experience refractory IMDC despite aggressive medical therapy and develops chronic IMDC. There is an inadequate understanding of the risk factors for chronic IMDC or its effects on the overall cancer outcome. We attempted to bridge this knowledge gap in a retrospective study of patients with advanced malignancies at The University of Texas MD Anderson Cancer Center (MDACC). 


\section{Methods}

\section{Patient Selection}

This retrospective study included consecutive patients at MDACC who were treated using ICI therapy from January 2018 through October 2019 and met the following inclusion criteria: (1) age $>18$ years, (2) established cancer diagnosis and undergoing ICI treatment, (3) grade $\geq 2$ diarrhea/colitis, (4) endoscopic and histologic evaluation at the time of IMDC onset, and (5) immunosuppressive treatment using steroids, infliximab, or vedolizumab. Patients with a prior diagnosis of inflammatory bowel disease (IBD), mesenteric ischemia, sepsis, concurrent gastrointestinal infection at the time of IMDC, and ICI resumption after IMDC during the study period were excluded. This study was approved by the MDACC Institutional Review Board with a waiver of patients' informed consent.

We defined chronic IMDC as a disease duration of $>3$ months (clinical symptoms or persistent histologic inflammation). The chronic IMDC group was divided into 3 subgroups: persistent symptomatic, recurrent, and persistent histologic IMDC. The persistent symptomatic group included patients with IMDC symptoms, despite medical treatment, until last follow-up. The recurrent group included those with symptoms of IMDC that recurred beyond 1 month after the resolution of the initial IMDC episode. The persistent histologic group had active histologic inflammation for $>3$ months on follow-up endoscopy, without clinical symptoms after medical treatment. Patients in the acute IMDC group had an overall disease duration $\leq 3$ months from the initial episode with sustained clinical remission while remaining off colitis treatments during the study period (with or without histology confirmation).

\section{Data Collection}

\section{Clinical and Oncology Data}

Baseline demographic data, oncology variables, IMDC features, colitis, and cancer outcomes were extracted from institutional electronic medical charts and pharmacy databases. Use of nonsteroidal anti-inflammatory drugs (NSAIDs) or proton pump inhibitors (PPIs) within 3 months before IMDC onset was reported. Cancer status at ICI initiation, IMDC onset, and last follow-up was evaluated based on RECIST guidelines, version 1.1. The severity of IMDC was graded according to CTCAE version 5.0. Clinical remission of symptoms was defined as a sustained resolution of symptoms to grade $\leq 1$ irAEs.

\section{Endoscopic and Histologic Evaluation}

Endoscopic findings included the presence of mucosal ulcerations, nonulcer inflammation, or normal appearance. Histologic patterns included acute or chronic active colitis, microscopic colitis, or normal patterns. Details of the features in each category have been previously described. ${ }^{11}$ Endoscopic and histologic remissions were defined as the resolution of ulceration/nonulcer inflammation endoscopically and the resolution of active histologic inflammation, respectively.

\section{Statistical Analysis}

SPSS Statistics, version 24.0 (IBM Corp) and GraphPad Prism (version 8.0) were used to perform the statistical analysis. Descriptive statistics of enrolled patients were calculated and presented as means, medians, standard deviations, and ranges. Fisher exact and chi-square tests were used to compare categorical variables. Continuous variables were compared using the Wilcoxon rank-sum or Kruskal-Wallis test. OS durations between groups were compared using the Kaplan-Meier method and the logrank test. A prognostic analysis was conducted using multivariate Cox regression analysis to measure OS. A logistic regression model was estimated with odds ratios (ORs) and 95\% confidence intervals to assess the risk factors of chronic IMDC and cancer progression. All statistical tests were 2-sided. Differences of $P<.05$ were considered statistically significant.

\section{Results}

\section{Patient Baseline Characteristics}

Our sample was composed of 88 patients, of whom 43 and 45 had chronic and acute IMDC, respectively. All patients discontinued ICIs due to IMDC. No new cancer diagnosis was reported during the follow-up period. $\mathrm{Pa}-$ tient selection diagram and baseline demographic characteristics are shown in supplemental eFigure 1 (available with this article at JNCCN.org) and Table 1. Median age was 65.5 years, and most patients were White and male. Genitourinary cancer $(47 \%)$ and melanoma $(23 \%)$ were the most common malignancies. ICI regimens included anti-CTLA-4 monotherapy ( $\mathrm{n}=15 ; 17 \%), \mathrm{PD}-1 / \mathrm{L} 1 \mathrm{mon}-$ otherapy $(n=46 ; 52 \%)$, and combination therapy $(n=27$; $31 \%)$. Dermatologic irAEs were most common, followed by IMDC. Patients with chronic IMDC had more frequent use of PPIs ( $91 \%$ vs $71 \% ; P=.030)$ and underwent more ICI cycles ( 10 vs $5 ; P=.033$ ) than those with acute IMDC over a longer duration (91 vs 42 days; $P=.017$; Table 1 ).

\section{IMDC-Related Characteristics}

Median duration of ICI treatment was 60.5 days (interquartile range [IQR], 21-160 days).The time to onset of chronic IMDC was delayed compared with time to onset of acute IMDC (104 vs 68 days; $P=.099$ ) (supplemental eTable 1). More than $50 \%$ of patients had grade $3 /$ 4 diarrhea and $35 \%$ had grade $3 / 4$ colitis, with a similar distribution in both groups. Patients with chronic IMDC 


\section{Table 1. Baseline Demographics}

\begin{tabular}{|c|c|c|c|c|}
\hline Characteristic & $\begin{array}{l}\text { Overall } \\
\text { n (\%) }\end{array}$ & $\begin{array}{c}\text { Chronic IMDC } \\
\text { (>3 mo) } \\
\text { n (\%) }\end{array}$ & $\begin{array}{c}\text { Acute IMDC } \\
(\leq 3 \mathrm{mo}) \\
\mathrm{n}(\%)\end{array}$ & $P$ Value \\
\hline Patients, $\mathrm{n}$ & 88 & 43 & 45 & \\
\hline Median age at time of IMDC diagnosis (IOR), y & $65.5(56-73)$ & $66(58-73)$ & $65(54-72)$ & .617 \\
\hline Male sex & $67(76)$ & $35(81)$ & $32(71)$ & .321 \\
\hline White race & $82(93)$ & $39(91)$ & $43(96)$ & .429 \\
\hline NSAID use & $63(72)$ & $27(63)$ & $36(80)$ & .099 \\
\hline PPI use & $71(81)$ & 39 (91) & 32 (71) & .030 \\
\hline Cancer type & & & & .363 \\
\hline Genitourinary & $41(47)$ & $18(42)$ & $23(51)$ & \\
\hline Melanoma & $20(23)$ & $12(28)$ & $8(18)$ & \\
\hline Hematologic & $2(2)$ & 1 (2) & $1(2)$ & \\
\hline Other & $4(5)$ & $3(7)$ & 1 (2) & \\
\hline Cancer stage & & & & .252 \\
\hline III & $14(16)$ & $9(21)$ & $5(11)$ & \\
\hline IV & 74 (94) & $34(79)$ & 40 (89) & \\
\hline ICl type & & & & .879 \\
\hline CTLA-4 & $15(17)$ & $8(19)$ & $7(16)$ & \\
\hline PD-1/L1 & $46(52)$ & $23(53)$ & $23(51)$ & \\
\hline Combination & 27 (31) & $12(28)$ & 15 (33) & \\
\hline
\end{tabular}

Abbreviations: ICl, immune checkpoint inhibitor; IMDC, immune-mediated diarrhea and colitis; IQR, interquartile range; NSAID, nonsteroidal anti-inflammatory drug; PPI, proton pump inhibitor.

had a longer duration of steroid use (80 vs 35 days; $P<.001)$ and more steroid tapering attempts (2 vs 1 ; $P<.001)$ than those with acute IMDC. We found that $84 \%$ of patients had endoscopic evidence of inflammation. However, no differences were found in endoscopic disease presentation and distribution between the 2 groups. All patients had an abnormal histology, with chronic active colitis and microscopic colitis being the predominant histologic patterns in the chronic group compared with the acute group (93\% vs $74 \% ; P=.049)$. Vedolizumab was the most commonly used SIT in the chronic group compared with the acute group (95\% vs $46 \% ; P<.001$ ), with $95 \%$ of patients in the chronic group requiring $\geq 3$ doses of SITs compared with $31 \%$ in the acute group $(P<.001)$. Patients with acute IMDC had higher rates of clinical remission ( $100 \%$ vs $74 \%$; $P<.001)$, endoscopic remission (88\% vs $47 \% ; P=.006)$, and histologic remission ( $71 \%$ vs $21 \%$; $P=.001$ ) than those with chronic IMDC. Among patients with refractory cases, $37 \%$ required a switch of SITs and $21 \%$ underwent compassionate fecal microbiota transplantation (FMT). There were no reported ICU admissions/deaths related to IMDC. Median duration from steroid or SIT use to histologic remission was 55 and 85 days, respectively $(P>.05)$.

Among patients with chronic IMDC, those with persistent symptoms had the longest duration of steroid therapy (160 vs 80 vs 64 days; $P<.001$ ) and the highest number of steroid tapering attempts ( 3 vs 2 vs $1 ; P=.001$ ) compared with the recurrent and persistent histologic groups, respectively (supplemental eTable 2).

\section{Risk Factors for Chronic IMDC}

PPI use (OR, 3.96; $P=.026)$; high ICI dose (OR, 1.06; $P=.056$ ); long duration of ICI treatment (OR, 1.01; 
$P=.053$ ), symptoms (OR, 1.05; $P<.001$ ), and hospitalization from initial episode (OR, 1.07; $P=.043)$; histologic features of chronic active colitis (OR, 4.8; $P=.025)$ or microscopic colitis (OR, 5.0; $P=.045)$; and delayed introduction of SIT for IMDC (OR, 1.06; $P=.047$ ) were identified as risk factors for chronic IMDC (Table 2). Histologic features of chronic active/microscopic colitis were significantly associated with chronic IMDC on multivariate logistic regression analysis (OR, 4.54; $P=.031$; Table 2).

\section{Risk Factors for Cancer Progression}

All patients had cancer progression at the time of ICI initiation. The proportion of patients with persistent cancer progression at the time of IMDC was significantly lower in the chronic group than in the acute group $(14 \%$ vs $33 \% ; P=.019$ ). At the last follow-up, the rate of cancer progression increased in both groups but was significantly lower in the chronic group (30\% vs $51 \%$; $P=.002$; Figure 1).

Univariate analysis revealed that chronic IMDC was associated with less risk of cancer progression (OR, $0.37 ; P=.028)$. Use of infliximab-based therapy (OR, 3.16; $P=.011)$, advanced cancer stage at baseline (OR, 11.05; $P=.024)$, and cancer progression status at the time of IMDC diagnosis (OR, 5.88; $P=.001$ ) were associated with a higher risk of cancer progression (Table 3 ). The association was reiterated per multivariate analysis between cancer progression at last follow-up and infliximabbased therapy (OR, 5.10; $P=.004$ ) and cancer progression status at the time of IMDC diagnosis (OR, 9.19; $P=.001$ ). Among the 3 subgroups of chronic IMDC, no particular subtype was associated with cancer progression $(P>.05)$.

\section{OS Analyses}

Median clinical follow-up duration in this study was 11 months (IQR, 7-20 months). Chronic IMDC was associated with longer OS $(P=.035$; Figure $2 \mathrm{~A})$. Longer OS was observed in patients who received $\geq 3$ doses of SIT than in those who received 1 to 2 doses $(P=.018$; Figure 2B). In subgroup analysis, patients with recurrent and persistent histologic IMDC had longer OS durations than those with persistent symptomatic IMDC ( $P=.036$ and $P=.041$, respectively; Figure $3 \mathrm{~A}, \mathrm{~B})$. However, OS duration did not differ between the recurrent and persistent histologic IMDC groups $(P=.778$; Figure 3C).

\section{Discussion}

Although ICI therapy has resulted in exceptional outcomes in treating advanced malignancies, its use is frequently limited by irAEs. ${ }^{12}$ There remains a knowledge gap regarding the long-term disease course and cancer

\begin{tabular}{|c|c|c|}
\hline Risk Factor & OR $(95 \% \mathrm{Cl})$ & $P$ Value \\
\hline \multicolumn{3}{|l|}{ Univariate analysis } \\
\hline Male sex & $1.78(0.65-4.85)$ & .261 \\
\hline White race & $0.45(0.08-2.61)$ & .453 \\
\hline Smoking status & $1.09(0.48-2.53)$ & .831 \\
\hline NSAID use & $0.42(0.16-1.10)$ & .077 \\
\hline PPI use & $3.96(1.18-13.34)$ & .026 \\
\hline \multicolumn{3}{|l|}{ Cancer stage } \\
\hline III & Ref & \\
\hline $\mathrm{IV}$ & $0.47(0.14-1.54)$ & .215 \\
\hline Overall duration of $\mathrm{ICl}$ treatment & $1.01(1.00-1.01)$ & .053 \\
\hline $\mathrm{ICl}$ dose & $1.06(0.99-1.13)$ & .056 \\
\hline \multicolumn{3}{|l|}{$\mathrm{ICl}$ type } \\
\hline PD-1/L1 & Ref & \\
\hline CTLA-4 & $1.29(0.41-4.04)$ & .667 \\
\hline Combination & $1.25(0.48-3.25)$ & .531 \\
\hline Duration from ICI to IMDC onset & $1.01(1.00-1.05)$ & .077 \\
\hline Duration of initial IMDC symptoms & $1.05(1.02-1.07)$ & $<.001$ \\
\hline Duration of hospitalization for initial IMDC & $1.07(1.00-1.13)$ & .043 \\
\hline \multicolumn{3}{|l|}{ Diarrhea grade } \\
\hline $1-2$ & Ref & \\
\hline $3-4$ & $1.27(0.52-3.11)$ & .596 \\
\hline \multicolumn{3}{|l|}{ Colitis grade } \\
\hline $1-2$ & Ref & \\
\hline $3-4$ & $0.79(0.33-1.91)$ & .609 \\
\hline \multicolumn{3}{|l|}{ Endoscopic presentation } \\
\hline Normal & Ref & \\
\hline Mucosal ulceration & $0.48(0.12-2.01)$ & .315 \\
\hline Nonulcerative inflammation & $0.27(0.08-0.98)$ & .265 \\
\hline \multicolumn{3}{|l|}{ Histologic features } \\
\hline Acute active colitis & Ref & \\
\hline Chronic active colitis & $4.8(1.22-18.93)$ & .025 \\
\hline Microscopic colitis & $5.0(1.04-24.03)$ & .045 \\
\hline Time from IMDC to first dose of SIT & $1.06(1.00-1.03)$ & .047 \\
\hline Additional non- $\mathrm{ICl}$ cancer treatment & $0.76(0.33-1.77)$ & .529 \\
\hline \multicolumn{3}{|l|}{ Multivariate analysis } \\
\hline Overall duration of $\mathrm{ICl}$ treatment & $1.08(0.98-1.12)$ & .103 \\
\hline $\begin{array}{l}\text { Histologic features of chronic active or } \\
\text { microscopic colitis }\end{array}$ & $4.54(1.15-17.94)$ & .031 \\
\hline Time from IMDC to first dose of SIT & $1.32(0.86-2.04)$ & .204 \\
\hline
\end{tabular}

Abbreviations: ICl, immune checkpoint inhibitor; IMDC, immune-mediated diarrhea and colitis; NSAID, nonsteroidal anti-inflammatory drug; OR, odds ratio; PPI, proton pump inhibitor; SIT, selective immunosuppressive therapy. 


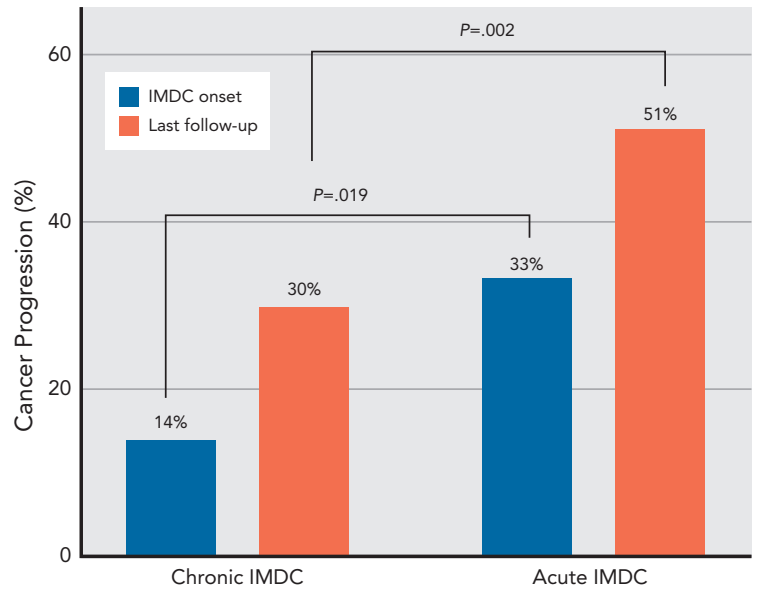

Figure 1. Cancer outcome stratified by IMDC duration. Abbreviation: IMDC, immune-mediated diarrhea and colitis.

outcomes of patients with IMDC. This study is the first focused analysis of the characteristics of chronic IMDC and their prognostic role in long-term cancer outcome and survival. We found that chronic IMDC can develop in patients who are exposed to higher dosages/longer durations of ICI therapy, have a delayed introduction of SIT, and have features of chronic active and microscopic colitis on histology. Chronic IMDC, a sign of prolonged ICI effect, is associated with beneficial cancer outcomes and OS.

Increasing evidence suggests that IMDC is predictive of improved OS. In particular, severe clinical symptoms and endoscopic presentations, ${ }^{12-14}$ accompanied by extensive organ toxicity, ${ }^{15}$ are associated with longer OS. However, prolonged IMDC duration as another feature of severe disease has never been studied. Therefore, follow-up endoscopic and histologic examination are not routinely recommended by current oncology guidelines. Although clinical remission remains the treatment target for IMDC in current practice, we found that a substantial number of patients had persistent histologic inflammation in the absence of clinical symptoms despite aggressive management, indicating that this target grossly underestimates the true incidence of lingering ICI toxicity at the microscopic level. The results of our analysis suggest the continuance of histologic features for up to 36.3 months after IMDC diagnosis. Fujii et $\mathrm{al}^{16}$ reported a stable cancer duration of 21 months, requiring the readministration of nivolumab after persistent IMDC in a patient with recurrent melanoma. Singh et $\mathrm{al}^{5}$ reported a patient with refractory IMDC that persisted for up to 8 months despite aggressive therapy.

Notably, clinical symptoms of IMDC do not always correlate with endoscopic or histologic inflammation. ${ }^{11}$ Because endoscopic and histologic remission are strongly associated with a lower IMDC recurrence, ${ }^{9}$ premature termination of IMDC treatment can be detrimental in patients with recurrent and refractory IMDC. In our study, $87.5 \%$ patients reached clinical remission, whereas a minority (23\%) had histologic remission at a median interval of approximately 2 months from the time of IMDC diagnosis. Furthermore, few patients had clinical and histologic recurrence despite initial histologic remission. This finding suggests the need for long-term endoscopic and histologic surveillance to predict longterm IMDC prognosis.

Previous studies have shown that the development of IMDC may reflect an enhanced, prolonged, and protective ICI effect against underlying cancer, resulting in significantly better OS and progression-free survival. ${ }^{12,13}$ Currently, introductory medical treatment for IMDC is corticosteroid therapy followed by SITs. However, the early introduction of SIT can result in higher efficacy against IMDC. ${ }^{9,17,18}$ In patients with refractory IMDC, the long-term use of SIT and frequent courses of steroid treatment are required. In our cohort, patients with refractory IMDC received up to 10 doses of vedolizumab or 5 doses of infliximab. Because the mechanism of action of ICIs relies on increasing antitumor immunity, it is possible that the use of systemic immunosuppression to treat irAEs could mitigate antineoplastic efficacy, leading to a decreased survival benefit. The association between secondary malignancies and the long-term use of tumor necrosis factor- $\alpha$ inhibitors was shown in a few retrospective studies of IBD. ${ }^{19-21}$ Some

\begin{tabular}{|c|c|c|}
\hline Characteristic & OR $(95 \% \mathrm{Cl})$ & $P$ Value \\
\hline Chronic IMDC (>3 mo) & $0.37(0.15-0.91)$ & .028 \\
\hline Duration of steroid administration & $0.99(0.99-1.01)$ & 178 \\
\hline Doses of SIT add-on & $0.69(0.54-0.91)$ & .005 \\
\hline \multicolumn{3}{|l|}{ Type of SIT } \\
\hline Vedolizumab & Ref & \\
\hline Infliximab and combination & $3.16(1.05-7.58)$ & .011 \\
\hline Overall duration of $\mathrm{ICI}$ treatment & $0.99(0.99-1.00)$ & .062 \\
\hline $\mathrm{ICl}$ dose & $0.93(0.87-1.01)$ & .065 \\
\hline \multicolumn{3}{|l|}{ ICI type } \\
\hline PD-1/L1 & Ref & 634 \\
\hline CTLA-4 & $0.89(1.02-3.25)$ & .962 \\
\hline Combination & $0.73(0.28-1.93)$ & .634 \\
\hline Cancer stage IV & $11.05(1.37-88.87)$ & .024 \\
\hline \multicolumn{3}{|l|}{ Cancer status at the time of IMDC onset } \\
\hline Cancer-free remission, stable, or response & Ref & \\
\hline Cancer progression & $5.88(0.98-17.33)$ & .001 \\
\hline
\end{tabular}

Abbreviations: $\mathrm{ICl}$, immune checkpoint inhibitor; IMDC, immune-mediated diarrhea and colitis; OR, odds ratio; SIT, selective immunosuppressive therapy. 
A

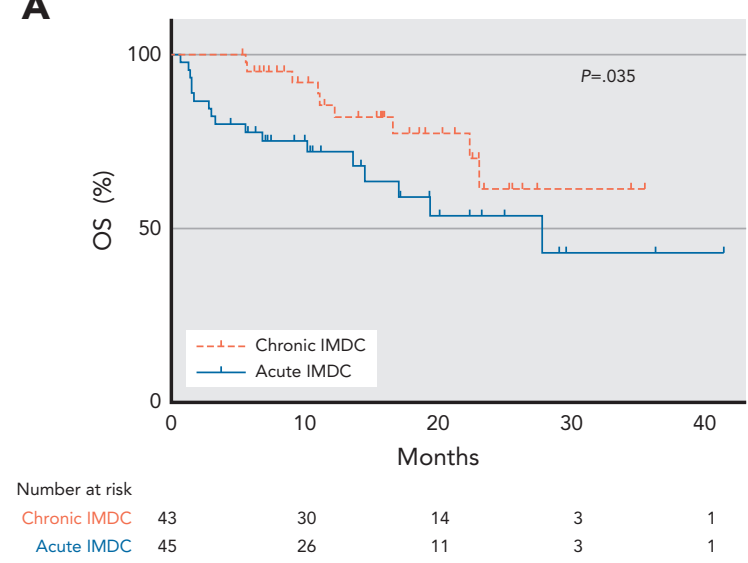

B

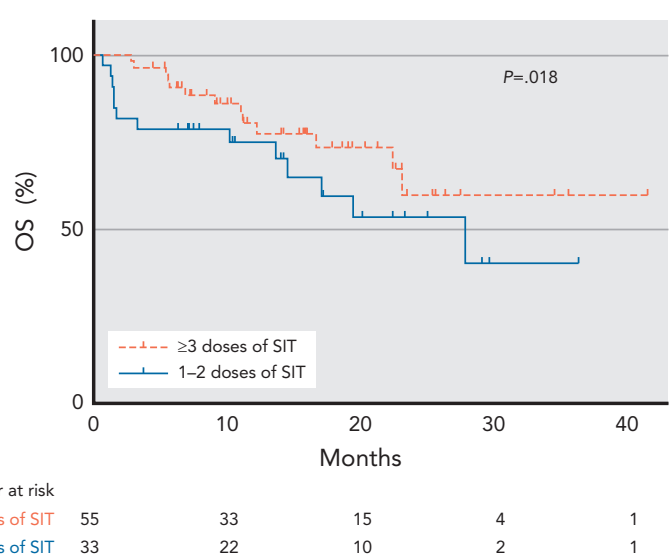

Figure 2. Kaplan-Meier curves showing OS duration between patients with (A) chronic (>3 months) and acute ( $\leq 3$ months) IMDC and (B) 1 to 2 doses of SIT (infliximab or vedolizumab) versus $\geq 3$ doses of SIT.

Abbreviations: IMDC, immune-mediated diarrhea and colitis; OS, overall survival; SIT, selective immunosuppressive therapy.

studies have suggested that certain immunosuppressive therapies lead to shorter OS and tumor progression..$^{22,23}$ Verheijden et $\mathrm{al}^{24}$ found that infliximab for steroidrefractory IMDC abrogated the survival advantage in the prospective nationwide Dutch Melanoma Treatment Registry. Other retrospective cohorts of patients with melanoma treated for irAEs using systemic immunosuppressants did not show a survival benefit compared with those who were not treated ${ }^{25,26}$ However, the confounding factors of these studies cannot be neglected.

In keeping with the findings of the studies mentioned earlier, we showed that although patients with chronic IMDC received significantly more SITs or steroid therapy, they had a better cancer response compared with patients with acute IMDC. Several factors should be considered. First, vedolizumab treatment constituted the bulk of the SITs used in our study, which may serve as a protective factor because it mitigates the antitumor effect of ICIs to a lesser extent than infliximab without increasing the risk of secondary malignancies in vulnerable patients. ${ }^{10}$ This practice pattern followed the MDACC institutional management algorithm: vedolizumab has equal priority to infliximab for IMDC treatment and is more favored when long-term use is anticipated. ${ }^{27}$ Second, approximately $50 \%$ of these patients resumed non-ICI anticancer therapy, which could have contributed to a longer OS. ${ }^{9}$ Third, a small group of patients underwent compassionate FMT for IMDC, which could have altered the cancer outcome. Prospective studies are needed to explore the relationship between immunosuppressant use and clinical outcomes.

There are various risk factors contributing to chronic IMDC. In our routine clinical practice, patients with active gastrointestinal symptoms or a history of colitis are commonly referred to gastroenterologists for endoscopic evaluation and risk assessment before ICI initiation to minimize colitis exacerbation. Given the increased risk of severe gastrointestinal irAEs secondary to ICI therapy conferred by preexisting IBD, ${ }^{28}$ it is particularly important to know that no patients in our sample bore this medical history. However, the histology feature of chronicity could be another contributing factor. Most patients with chronic IMDC in our sample were histologically diagnosed with chronic active or microscopic colitis, both of which overlap with IBD and non-ICI-related microscopic colitis. Longterm follow-up is necessary to determine whether these acquired histologic types would behave like de novo types. Similar to Crohn colitis, large deep colonic ulcerations from IMDC are associated with a steroid-refractory disease course and are likely to persist long-term despite successful initial treatment. ${ }^{29}$ ICI-related microscopic colitis also has a more aggressive disease course than the non-ICI type, which requires potent immunosuppression. ${ }^{30}$ We postulate that acute histologic features may trigger symptoms earlier than chronic active or microscopic colitis, prompting timely evaluation and treatment. Late onset of clinical symptoms delays diagnosis and treatment of chronic IMDC and results in a prolonged disease course. The significance of histologic conversion from acute to chronic IMDC requires further elucidation.

Interestingly, PPI use was identified as a risk factor for chronic IMDC after an initial diagnosis. No prior studies have shown a correlation between chronic IMDC and PPI use. We hypothesize that our findings resulted from the known influence of this class of routinely used medications on the gut microbiota ${ }^{31}$ : specifically, the increased risk of enteric infections, bacterial translocation, ${ }^{32}$ and the pathogenesis of microscopic colitis (one of the histologic variants of IMDC). ${ }^{33,34}$ Furthermore, acid 
A

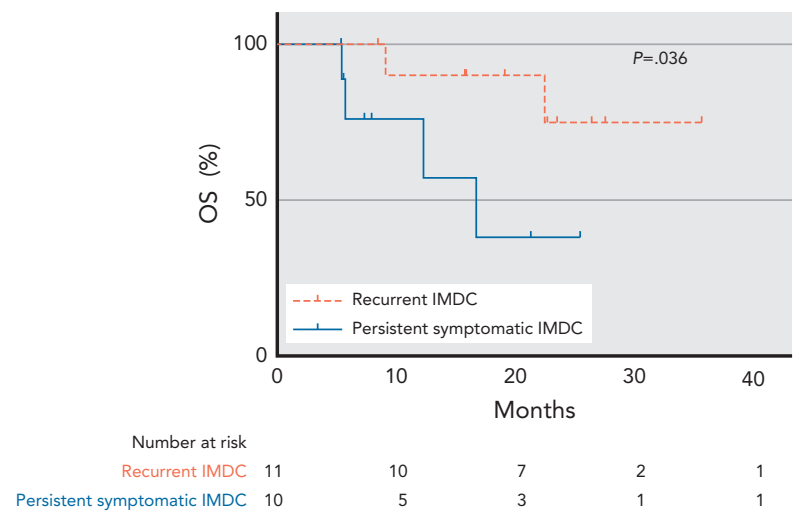

B

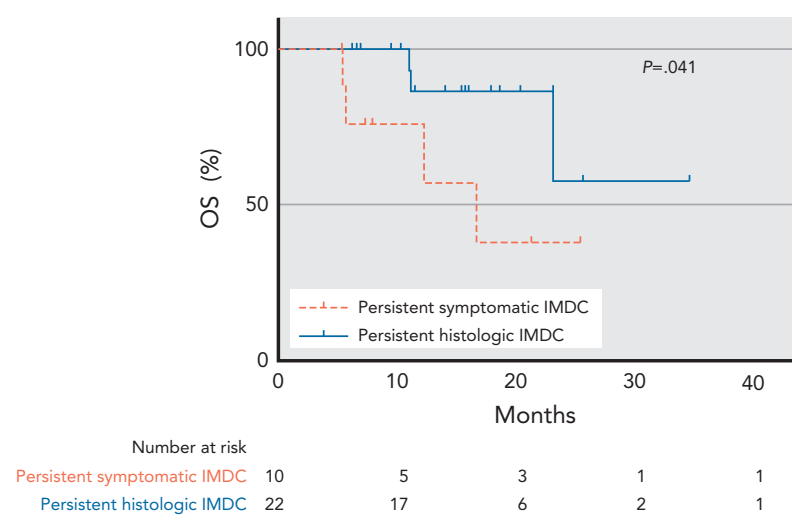

C

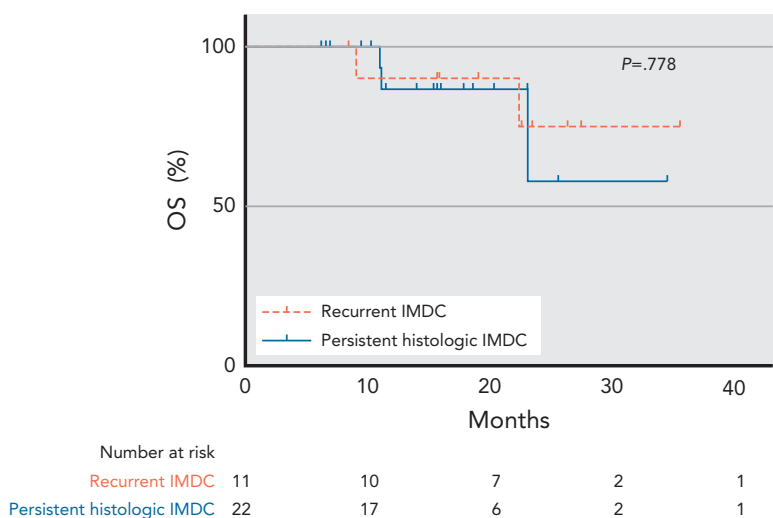

Figure 3. Kaplan-Meier curves showing OS duration between patients with (A) persistent symptomatic and recurrent IMDC; $(B)$ persistent symptomatic and persistent histologic IMDC; and (C) recurrent and persistent histologic IMDC.

Abbreviations: IMDC, immune-mediated diarrhea and colitis; OS, overall survival.

suppression-related gut dysbiosis from PPI may be exacerbated by NSAID-related adverse effects. ${ }^{34,35}$ Therefore, proper indications should continue to be observed when prescribing or continuing PPIs in patients with IMDC.
Although chronic IMDC is highly predictive of a prolonged ICI effect and thereby of beneficial cancer outcomes, management of patients with refractory disease is challenging with limited therapeutic options. The gut microbiome has been shown to play a critical role in modulating the safety of ICI therapy, as noted in recent studies regarding the impact of antibiotics on IMDC incidence and treatment response. ${ }^{36,37}$ Gut dysbiosis is implicated in the development of gastrointestinal irAEs via varied pathways, and influences the response to immunotherapy and cancer outcomes. ${ }^{38-40}$ Microbiota alterations after ICI therapy contribute to a sustainable effect in prolonging colitis while maintaining a response to cancer. Although data on the long-term cancer outcomes of FMT are lacking, evidence suggests that modulation of the gut microbiome via FMT can result in significant and rapid improvement of refractory and chronic IMDC. ${ }^{10}$ It is yet to be determined whether modulation of the gut microbiome aids in the prolonged therapeutic effects of ICI and thereby improves OS in these patients with cancer.

Although we have evidence to suggest that resuming ICIs in patients with IMDC is associated with an approximately $34 \%$ overall risk of exacerbation, ${ }^{5}$ we hypothesize that this risk is substantially higher in those with chronic IMDC. We propose the need for a tool to determine the optimal timing of ICI resumption, estimate the risk of IMDC exacerbation, and measure the utility of adjunct SIT therapy with long-term ICI treatment.

Our study has several limitations. First, this retrospective study with a small sample size limited our power to perform an adequate subgroup analysis. Second, the absence of gastrointestinal symptoms before ICI initiation did not prompt routine endoscopy evaluation; therefore, preexisting mild colitis could have been underdiagnosed and confounded our analysis. Third, given the lack of standard guidelines for the long-term surveillance of IMDC, not every patient underwent repeat endoscopy, which could have led to a bias regarding disease outcome. Finally, the short median follow-up period of 11 months may limit the accurate representation of the long-term outcomes of IMDC and cancer.

\section{Conclusions}

Chronic IMDC can develop among patients with more aggressive disease and chronic histologic features. This condition should be considered a distinct irAE reflective of prolonged ICI effect and thus beneficial cancer treatment outcomes. Given the likelihood of underestimating the true incidence of prolonged ICI toxicity at the microscopic level by using clinical remission as the treatment target, optimization of outcome measures is strongly indicated. Future larger-scale studies are warranted to clarify the predictive value of histologic features in chronic IMDC and outcomes related to cancer treatment. 


\section{Acknowledgments}

Medical editing of this article was provided by Scientific Publications, Research Medical Library, The University of Texas MD Anderson Cancer Center.

Submitted June 25, 2020; final revision received August 11, 2020 accepted for publication August 31, 2020.

Published online December 14, 2020.

Author contributions: Study concept and design: Thomas, Y. Wang. Data collection: Zou. Conduct and interpretation of analysis: Zou. Project design: Abu-Sbeih, Ma, Peng. Interpretation of results: Thomas, Y. Wang. Preservation of data accuracy and integrity at all stages: Thomas, Y. Wang. Biostatistical analysis: Qiao. Manuscript preparation: Thomas, Y. Wang, Zou. Critical revision: Abu-Sbeih, Ma, Peng, J. Wang, Shah, Glitza Oliva, Piha-Paul, Thompson, Zhang.

Disclosures: Dr. Piha-Paul has disclosed that she receives grant/research support (through her institution) from AbbVie, Inc.; ABM Therapeutics, Inc.; Acepodia, Inc; Alkermes; Aminex Therapeutics; Amphivena Therapeutics, Inc.; BioMarin Pharmaceutical, Inc; Boehringer Ingelheim; Bristol-Myers Squibb;
Cerulean Pharma, Inc.; Chugai Pharmaceutical Co., Ltd: Curis, Inc.; Daichi Sanko; Eli Lilly; ENB Therapeutics; Five Prime Therapeutics; Gene Quantum; Genmab A/S; GlaxoSmithKline; Helix BioPharma Corp.; Incyte Corporation; Jacobio Pharmaceuticals Co., Ltd.; Medimmune, LLC.; Medivation, Inc.; Merck Sharp \& Dohme Corp.; Novartis Pharmaceuticals; Pieris Pharmaceuticals, Inc. Pfizer; Principia Biopharma, Inc.; Puma Biotechnology, Inc.; Rapt Therapeutics, Inc.; Seattle Genetics; Silverback Therapeutics; Taiho Oncology; Tesaro, Inc.; and TransThera Bio. The remaining authors have disclosed that they have not received any financial consideration from any person or organization to support the preparation, analysis, results, or discussion of this article.

Funding: Dr. Piha-Paul is the recipient of an $\mathrm{NCl} / \mathrm{NIH}$ Core Grant (P30CA016672 - CCSG shared resources).

Disclaimers: Ethics approval for this study was granted by The University of Texas MD Anderson Cancer Center Institutional Review Board (PA18-0472). Patient consent was waived for this study. The datasets used and analyzed in this study are available from the corresponding author upon reasonable request.

Correspondence: Yinghong Wang, MD, PhD, Department of

Gastroenterology, Hepatology, and Nutrition, The University of Texas MD Anderson Cancer Center, Unit 1466, 1515 Holcombe Boulevard, Houston, TX 77030. Email: ywang59@mdanderson.org

\section{References}

1. Darvin P, Toor SM, Sasidharan Nair V et al. Immune checkpoint inhibitors: recent progress and potential biomarkers. Exp Mol Med 2018;50:1-11.

2. Bayle A, Khettab M, Lucibello F, et al. Immunogenicity and safety of influenza vaccination in cancer patients receiving checkpoint inhibitors targeting PD-1 or PD-L1. Ann Oncol 2020;31:959-961.

3. Abril-Rodriguez G, Ribas A. SnapShot: immune checkpoint inhibitors. Cancer Cell 2017;31:848-848.e1.

4. Marin-Acevedo JA, Soyano AE, Dholaria B, et al. Cancer immunotherapy beyond immune checkpoint inhibitors. J Hematol Oncol 2018;11:8.

5. Singh BP, Marshall JL, He AR. Workup and management of immunemediated colitis in patients treated with immune checkpoint inhibitors. Oncologist 2020;25:197-202

6. Bellaguarda E, Hanauer S. Checkpoint inhibitor-induced colitis. Am J Gastroenterol 2020;115:202-210.

7. Abu-Sbeih H, Ali FS, Naqash AR, et al. Resumption of immune checkpoint inhibitor therapy after immune-mediated colitis. J Clin Oncol 2019;37: 2738-2745.

8. Abu-Sbeih H, Ali FS, Wang Y. Immune-checkpoint inhibitors induced diarrhea and colitis: a review of incidence, pathogenesis and management. Curr Opin Gastroenterol 2020;36:25-32.

9. Abu-Sbeih H, Ali FS, Wang $X$, et al. Early introduction of selective immunosuppressive therapy associated with favorable clinical outcomes in patients with immune checkpoint inhibitor-induced colitis. J Immunother Cancer 2019;7:93.

10. Som A, Mandaliya R, Alsaadi D, et al. Immune checkpoint inhibitor-induced colitis: a comprehensive review. World J Clin Cases 2019;7:405-418.

11. Wang $Y$, Abu-Sbeih H, Mao E, et al. Endoscopic and histologic features of immune checkpoint inhibitor-related colitis. Inflamm Bowel Dis 2018;24: 1695-1705.

12. Wang $Y$, Abu-Sbeih $H$, Mao E, et al. Immune-checkpoint inhibitor-induced diarrhea and colitis in patients with advanced malignancies: retrospective review at MD Anderson. J Immunother Cancer 2018:6:37.

13. Abu-Sbeih H, Ali FS, Qiao W, et al. Immune checkpoint inhibitor-induced colitis as a predictor of survival in metastatic melanoma. Cancer Immunol Immunother 2019;68:553-561.

14. Wang $Y$, Wiesnoski DH, Helmink BA, et al. Fecal microbiota transplantation for refractory immune checkpoint inhibitor-associated colitis. Nat Med 2018;24:1804-1808.

15. Abu-Sbeih $\mathrm{H}$, Tang $\mathrm{T}$, Ali FS, et al. The impact of immune checkpoint inhibitor-related adverse events and their immunosuppressive treatment on patients' outcomes. J Immunother Precis Oncol 2018;1:7-18.

16. Fujii $Y$, Nishikawa $Y$, Nomura $M$, et al. Readministration of nivolumab after persistent immune-related colitis in a patient with recurrent melanoma. Intern Med 2018;57:1173-1176.

17. Johnson DH, Zobniw CM, Trinh VA, et al. Infliximab associated with faster symptom resolution compared with corticosteroids alone for the management of immune-related enterocolitis. J Immunother Cancer 2018;6:103

18. Abu-Sbeih H, Ali FS, Alsaadi D, et al. Outcomes of vedolizumab therapy in patients with immune checkpoint inhibitor-induced colitis: a multi-center study. J Immunother Cancer 2018;6:142.

19. van den Heuvel TR, Wintjens DS, Jeuring SF, et al. Inflammatory bowe disease, cancer and medication: cancer risk in the Dutch populationbased IBDSL cohort. Int J Cancer 2016;139:1270-1280.

20. Axelrad JE, Lichtiger S, Yajnik V. Inflammatory bowel disease and cancer: the role of inflammation, immunosuppression, and cancer treatment. World J Gastroenterol 2016;22:4794-4801.

21. Taleban S, Elquza E, Gower-Rousseau C, et al. Cancer and inflammatory bowel disease in the elderly. Dig Liver Dis 2016;48:1105-1111.

22. Scott SC, Pennell NA. Early use of systemic corticosteroids in patients with advanced NSCLC treated with nivolumab. J Thorac Oncol 2018:13: 1771-1775.

23. Esfahani K, Miller WH Jr. Reversal of autoimmune toxicity and loss of tumor response by interleukin-17 blockade. N Engl J Med 2017;376: 1989-1991.

24. Verheijden RJ, May AM, Blank CU, et al. Association of anti-TNF with decreased survival in steroid refractory ipilimumab and anti-PD1-treated patients in the Dutch Melanoma Treatment Registry. Clin Cancer Res 2020;26:2268-2274

25. Horvat TZ, Adel NG, Dang TO, et al. Immune-related adverse events, need for systemic immunosuppression, and effects on survival and time to treatment failure in patients with melanoma treated with ipilimumab at Memorial Sloan Kettering Cancer Center. J Clin Oncol 2015;33: 3193-3198.

26. Arriola E, Wheater M, Karydis I, et al. Infliximab for IPILIMUMAB-related colitis-letter. Clin Cancer Res 2015;21:5642-5643.

27. The University of Texas MD Anderson Cancer Center. Evaluation and management of suspected immune-mediated colitis/diarrhea. Accessed September 10, 2020. Available at: https://www.mdanderson.org/content/dam/ mdanderson/documents/for-physicians/algorithms/clinical-management/ clin-management-immune-mediated-colitis-web-algorithm.pdf

28. Abu-Sbeih H, Faleck DM, Ricciuti B, et al. Immune checkpoint inhibitor therapy in patients with preexisting inflammatory bowel disease. J Clin Oncol 2020;38:576-583.

29. Abu-Sbeih H, Ali FS, Luo W, et al. Importance of endoscopic and histological evaluation in the management of immune checkpoint inhibitorinduced colitis. J Immunother Cancer 2018;6:95.

30. Choi K, Abu-Sbeih H, Samdani R, et al. Can immune checkpoint inhibitors induce microscopic colitis or a brand new entity? Inflamm Bowel Dis 2019; 25:385-393.

31. Freedberg DE, Toussaint NC, Chen SP, et al. Proton pump inhibitors alter specific taxa in the human gastrointestinal microbiome: a crossover trial. Gastroenterology 2015;149:883-885.e9

32. Ducarmon QR, Zwittink RD, Hornung BVH, et al. Gut microbiota and colonization resistance against bacterial enteric infection. Microbiology 
and molecular biology reviews. Microbiol Mol Biol Rev 2019;83: e00007-00009.

33. Bonderup OK, Nielsen GL, Dall M, et al. Significant association between the use of different proton pump inhibitors and microscopic colitis: a nationwide Danish case-control study. Aliment Pharmacol Ther 2018;48: 618-625.

34. Verhaegh BP, de Vries F, Masclee AA, et al. High risk of drug-induced microscopic colitis with concomitant use of NSAIDs and proton pump inhibitors. Aliment Pharmacol Ther 2016;43:1004-1013.

35. Rogers MAM, Aronoff DM. The influence of non-steroidal antiinflammatory drugs on the gut microbiome. Clin Microbiol Infect 2016;22: 178.e1-178.e9.

36. Becattini S, Taur Y, Pamer EG. Antibiotic-induced changes in the intestinal microbiota and disease. Trends Mol Med 2016;22:458-478.
37. Abu-Sbeih $\mathrm{H}$, Herrera LN, Tang $\mathrm{T}$, et al. Impact of antibiotic therapy on the development and response to treatment of immune checkpoint inhibitor-mediated diarrhea and colitis. J Immunother Cancer $2019 ; 7: 242$

38. Chaput $\mathrm{N}$, Lepage $\mathrm{P}$, Coutzac $\mathrm{C}$, et al. Baseline gut microbiota predicts clinical response and colitis in metastatic melanoma patients treated with ipilimumab. Ann Oncol 2017;28:1368-1379.

39. Matson V, Fessler J, Bao R, et al. The commensal microbiome is associated with anti-PD-1 efficacy in metastatic melanoma patients. Science 2018; 359:104-108.

40. Routy B, Le Chatelier E, Derosa L, et al. Gut microbiome influences efficacy of PD-1-based immunotherapy against epithelial tumors. Science 2018;359:91-97.

\section{Explore Oncology From Every Angle}

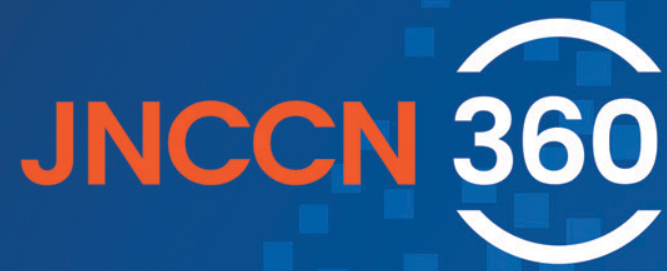

\section{The Hub for Disease-Specific Clinical Information}

> JNCCN Spotlights: exclusive commentary about current therapies

Video interviews with experts
Links to professional and patient resources

Summaries of news and medical literature
JNCCN360.org
\$ARBORSIDE

The nexus of knowledge
National Comprehensive Network 
Supplemental online content for:

\section{Association of Chronic Immune-Mediated Diarrhea and Colitis With Favorable Cancer Response}

Fangwen Zou, MD; Hamzah Abu-Sbeih, MD; Weijie Ma, MD; Yuanzun Peng, BA; Wei Qiao, PhD; Jianbo Wang, MD, PhD; Amishi Y. Shah, MD; Isabella C. Glitza Oliva, MD, PhD; Sarina A. Piha-Paul, MD; John A. Thompson, MD; Hao Chi Zhang, MD; Anusha S. Thomas, MD; and Yinghong Wang, MD, PhD

J Natl Compr Canc Netw 2021;19(6):700-708

eFigure 1: Flow Diagram of Patient Selection

eTable 1: Baseline Immune-Mediated Diarrhea and Colitis Characteristics Stratified by Disease Duration eTable 2: Characteristics of Patient Subgroups With Chronic Immune-Mediated Diarrhea and Colitis 


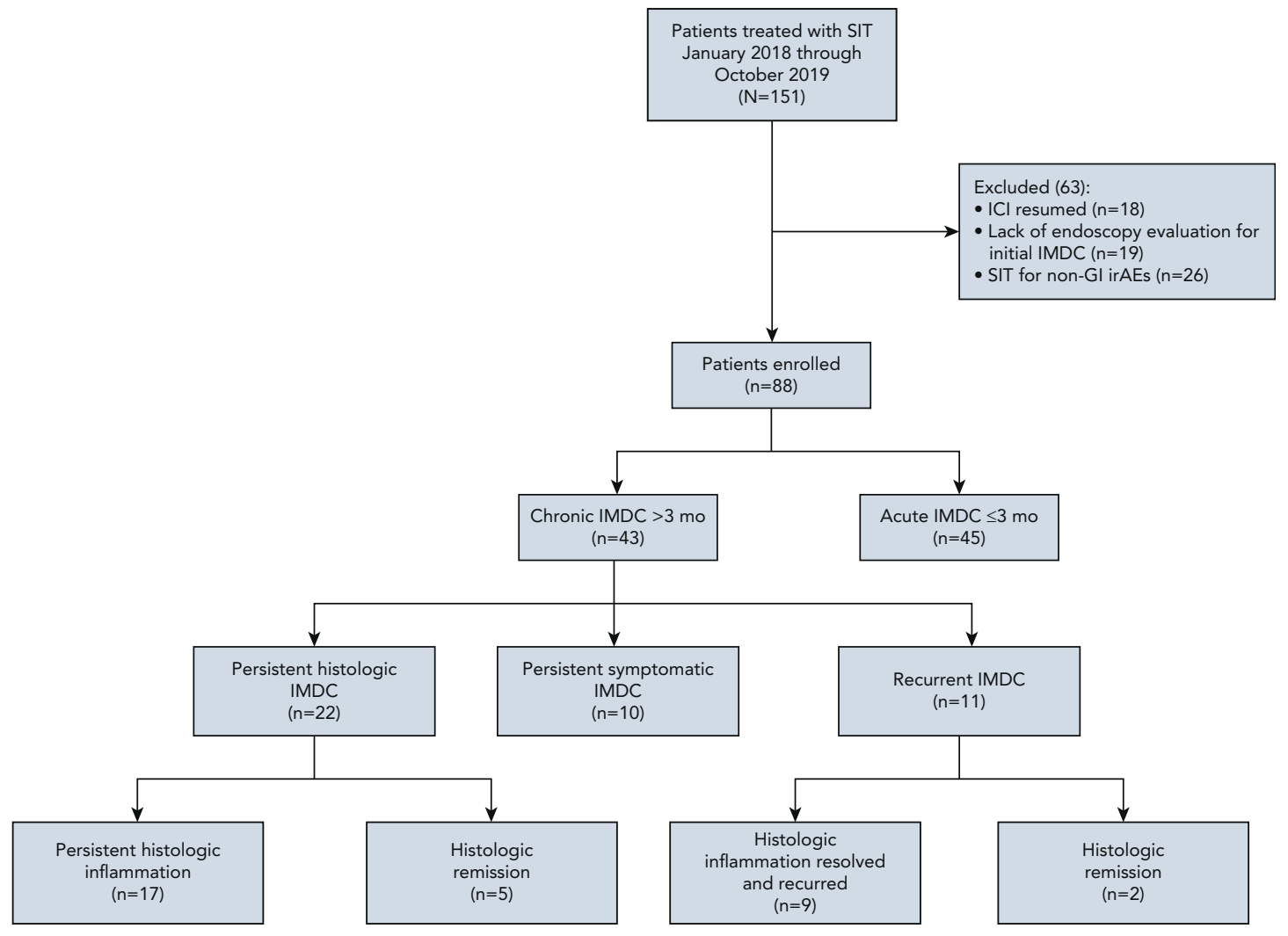

eFigure 1. Flow diagram of patient selection.

Abbreviations: Gl, gastrointestinal; ICl, immune checkpoint inhibitor; IMDC, immune-mediated diarrhea and colitis; irAEs, immune-related adverse effects; SIT, selective immunosuppressive therapy. 


\begin{tabular}{|c|c|c|c|}
\hline Characteristic & $\begin{array}{c}\text { Chronic IMDC } \\
\text { (>3 mo) } \\
\text { n (\%) }\end{array}$ & $\begin{array}{c}\text { Acute IMDC } \\
(\leq 3 \mathrm{mo}) \\
\mathrm{n}(\%)\end{array}$ & $\begin{array}{c}P \\
\text { Value }\end{array}$ \\
\hline Patients, $\mathrm{n}$ & 43 & 45 & \\
\hline $\begin{array}{l}\text { Median days from } \mathrm{ICl} \text { to } \\
\mathrm{IMDC} \text { onset (IQR), } n=88\end{array}$ & $104(46-220)$ & $68(46-116.5)$ & .099 \\
\hline $\begin{array}{l}\text { Median days of IMDC (IQR), } \\
\mathrm{n}=88\end{array}$ & $64(30-105)$ & $31(20.5-43)$ & $<.001$ \\
\hline Colitis grade & & & .927 \\
\hline $1-2$ & $29(67)$ & $28(62)$ & \\
\hline $3-4$ & $14(33)$ & $17(38)$ & \\
\hline Diarrhea grade & & & .746 \\
\hline $1-2$ & $13(30)$ & $16(36)$ & \\
\hline $3-4$ & $30(70)$ & $29(64)$ & \\
\hline Endoscopic presentation & & & .100 \\
\hline Mucosal ulceration & $12(28)$ & $10(22)$ & \\
\hline $\begin{array}{l}\text { Nonulcerative } \\
\text { inflammation }\end{array}$ & $21(49)$ & $31(69)$ & \\
\hline Normal & $10(23)$ & $4(9)$ & \\
\hline Disease location on endoscopy & & & .384 \\
\hline Colon & $30(70)$ & $36(80)$ & \\
\hline Small bowel & $1(2)$ & $0(0)$ & \\
\hline Both locations & $12(28)$ & $9(20)$ & \\
\hline Histologic features & & & .049 \\
\hline Acute active colitis & $3(7)$ & $12(27)$ & \\
\hline Chronic active colitis & $30(70)$ & $25(56)$ & \\
\hline Microscopic colitis & $10(23)$ & $8(18)$ & \\
\hline $\begin{array}{l}\text { Median days of steroids (IQR), } \\
n=83\end{array}$ & $80(57-125)$ & $35(25-54.5)$ & $<.001$ \\
\hline $\begin{array}{l}\text { Median number of steroid } \\
\text { tapering attempts (IQR) }\end{array}$ & $2(1-2)$ & $1(0-1)$ & $<.001$ \\
\hline Type of SIT & & & $<.001$ \\
\hline Vedolizumab & $26(60)$ & $19(42)$ & \\
\hline Infliximab & $2(2)$ & $24(53)$ & \\
\hline Combination & $15(35)$ & $2(4)$ & \\
\hline
\end{tabular}

\begin{tabular}{|c|c|c|c|}
\hline Characteristic & $\begin{array}{c}\text { Chronic IMDC } \\
(>3 \mathrm{mo}) \\
\text { n (\%) }\end{array}$ & $\begin{array}{c}\text { Acute IMDC } \\
(\leq 3 \mathrm{mo}) \\
\mathrm{n}(\%)\end{array}$ & $\begin{array}{c}P \\
\text { Value }\end{array}$ \\
\hline $\begin{array}{l}\text { Median days from IMDC to } \\
\text { first dose of SIT (IQR), n=88 }\end{array}$ & $13(7-38)$ & $12(6.5-24.5)$ & .231 \\
\hline Infliximab, $n=42$ & $14.5(9-41)$ & $17.5(12-35)$ & \\
\hline Vedolizumab, $n=46$ & $13(3-42.5)$ & $9(4.5-20)$ & \\
\hline Number of doses of SIT & & & $<.001$ \\
\hline $1-2$ & $2(5)$ & $31(69)$ & \\
\hline$\geq 3$ & $41(95)$ & $14(31)$ & \\
\hline $\begin{array}{l}\text { Repeat endoscopy after } \\
\text { medical treatment, } n=55\end{array}$ & $38(88)$ & $17(38)$ & $<.001$ \\
\hline Change in IMDC treatment & & & $<.001$ \\
\hline Change of SITa & $16(37)$ & $2(5)$ & \\
\hline FMT & $9(21)$ & $0(0)$ & \\
\hline \multicolumn{4}{|l|}{ IMDC outcome } \\
\hline Hospitalization & $32(74)$ & $35(78)$ & .804 \\
\hline $\begin{array}{l}\text { Median days of } \\
\text { hospitalization (IQR), } n=69\end{array}$ & $9(6-13)$ & $7(4-10)$ & .177 \\
\hline Clinical remission & $32(74)$ & $45(100)$ & $<.001$ \\
\hline Endoscopic remission ${ }^{b}$ & $18(47)$ & $15(88)$ & .006 \\
\hline Histologic remission ${ }^{c}$ & $8(21)$ & $12(71)$ & .001 \\
\hline All-cause death & $9(21)$ & $17(38)$ & .104 \\
\hline
\end{tabular}

Abbreviations: FMT, fecal microbiota transplantation; ICl, immune checkpoint inhibitor; IMDC, immune-mediated diarrhea and colitis; IQR, interquartile range; SIT, selective immunosuppressive therapy.

${ }^{a}$ Change of nonsteroid immunosuppressant includes infliximab change to vedolizumab, vedolizumab change to infliximab, or infliximab or vedolizumab change to ustekinumab.

bThe denominator of endoscopic remission was patients with repeat endoscopy.

cThe denominator of histologic remission was patients with repeat endoscopy. 


\section{eTable 2. Characteristics of Patient Subgroups With Chronic IMDC ( $n=43)$}

\begin{tabular}{|c|c|c|c|c|}
\hline Characteristic & $\begin{array}{c}\text { Persistent } \\
\text { Symptomatic IMDC } \\
\mathbf{n}(\%)\end{array}$ & $\begin{array}{c}\text { Recurrent IMDC } \\
\text { n (\%) }\end{array}$ & $\begin{array}{c}\text { Persistent } \\
\text { Histologic IMDC } \\
\mathbf{n}(\%)\end{array}$ & $P$ Value \\
\hline Patients, $\mathrm{n}$ & 10 & 11 & 22 & \\
\hline Cancer type & & & & .253 \\
\hline Genitourinary & $5(50)$ & $4(36)$ & $9(41)$ & \\
\hline Melanoma & $3(30)$ & $3(27)$ & $6(27)$ & \\
\hline Head and neck & $1(10)$ & $1(9)$ & $2(9)$ & \\
\hline Gastrointestinal & $0(0)$ & $1(9)$ & $1(5)$ & \\
\hline Hematologic & $1(10)$ & $0(0)$ & $0(0)$ & \\
\hline Other & $0(0)$ & $1(9)$ & $2(9)$ & \\
\hline Cancer stage & & & & .255 \\
\hline CTLA-4 & $2(20)$ & $1(9)$ & $5(23)$ & \\
\hline PD-1/L1 & $4(40)$ & $8(73)$ & $11(50)$ & \\
\hline Combination & $4(40)$ & $2(18)$ & $6(27)$ & \\
\hline Median dose of ICI (IQR) & $3.5(1-6)$ & $8(6-26)$ & $3.5(2-15)$ & .564 \\
\hline Diarrhea grade & & & & .455 \\
\hline $1-2$ & $4(40)$ & $8(73)$ & $17(77)$ & \\
\hline $3-4$ & $6(60)$ & $3(27)$ & $5(23)$ & \\
\hline Colitis grade & & & & .200 \\
\hline $1-2$ & $1(10)$ & $3(27)$ & $9(41)$ & \\
\hline Acute active colitis & $0(0)$ & $1(9.09)$ & $2(9)$ & \\
\hline Chronic active colitis & $9(90)$ & $6(55)$ & $15(68)$ & \\
\hline Microscopic colitis & $1(10)$ & $4(36)$ & $5(23)$ & \\
\hline Median overall days of steroids (IQR), $n=83$ & $160(113-205)$ & 80 (59-98) & $64(43-81)$ & $<.001$ \\
\hline $\begin{array}{l}\text { Median number of steroid tapering attempts } \\
\text { before SIT use (IQR) }\end{array}$ & $3(2-3)$ & $2(1-2)$ & $1(0-2)$ & .001 \\
\hline
\end{tabular}

Abbreviations: ICI, immune checkpoint inhibitor; IMDC, immune-mediated diarrhea and colitis; IQR, interquartile range; SIT, selective immunosuppressive therapy. 